Supporting Information

\title{
Lateral and Normal Capillary Force Evolution of a Reciprocating Liquid Bridge
}

Qingrui Song, Kun Liu, Wei Sun, Rongxin Chen, Jiawei Ji, Yunlong Jiao, Tianyan Gao, Jiaxin Ye*

Institute of Tribology

School of Mechanical Engineering

Hefei University of Technology

Hefei, Anhui,

China 


\section{Linear fitting of the force signal offsets in the steady state period}
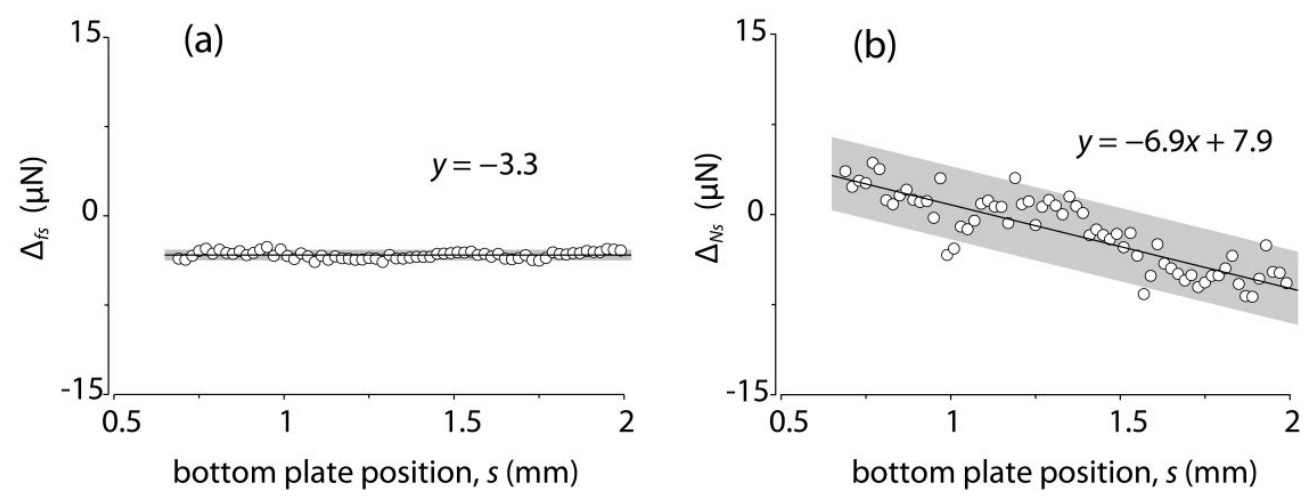

Fig. S1. (a) The offset of the lateral adhesion force, $\Delta_{f s}$, and (b) the offset of the normal adhesion force, $\Delta_{N s}$, during the steady-state sliding plotted against the bottom plate position. Both offsets could be fitted with linear functions based on Eq. 14 and Eq. 18.

\section{Evolution of the liquid bridge lengths and widths in the experiment}

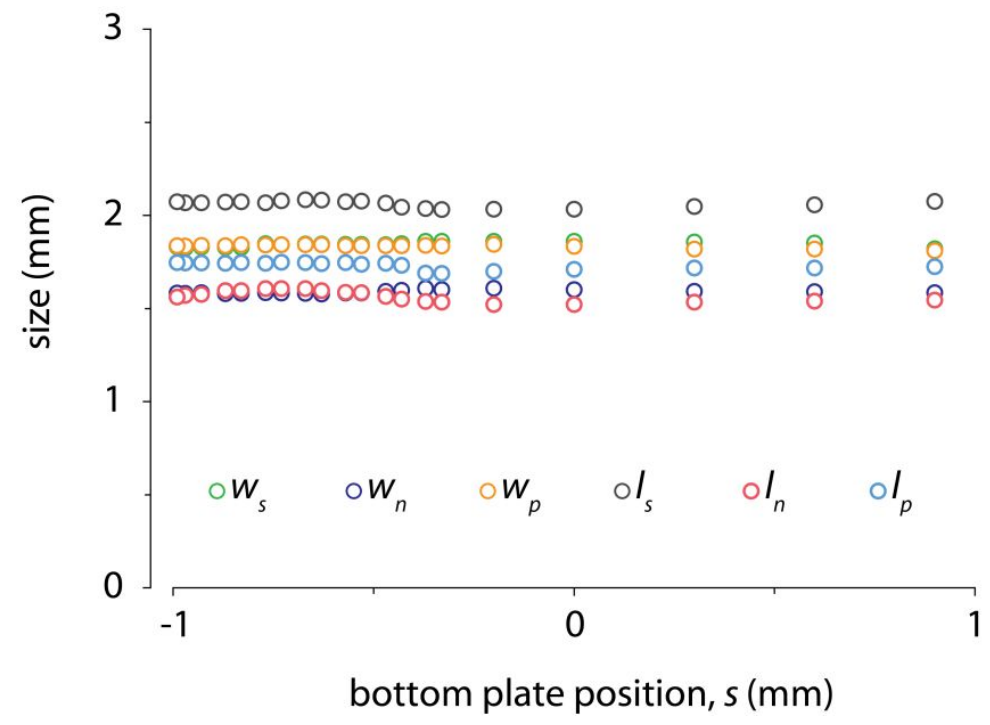

Fig. S2. Lengths and widths of the water bridge plotted against the bottom plate position during the reciprocated sliding experiment in this study. The relevant lengths and widths were measured in real time by in-situ observation and varied less than $5 \%$ during the sliding. 


\section{Pretreatment for the application of the modified wedge method}

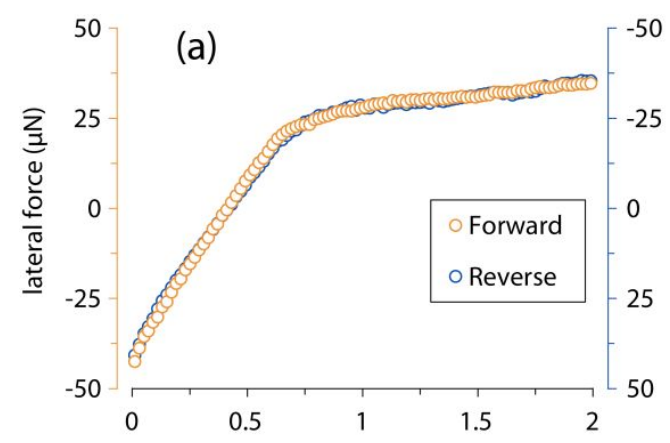

displacement from velocity turning point $(\mathrm{mm})$

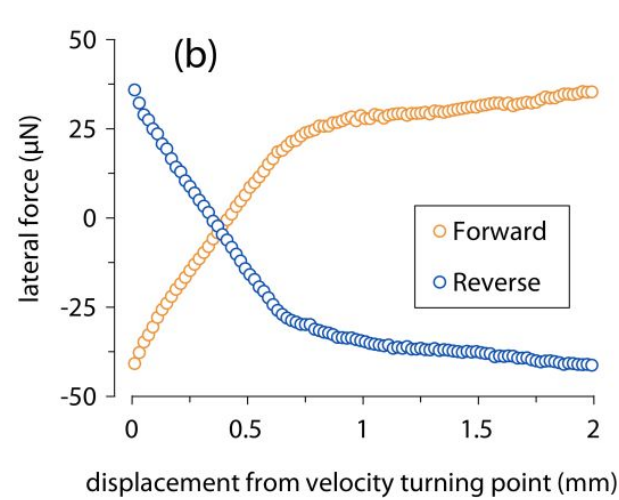

Fig. S3. Forward and reverse lateral forces plotted against the displacement from the velocity turning points. (a) The overlap between the forward and reverse data suggested that the reciprocal shear behavior of the liquid bridge was symmetric, which was in accordance with the assumption of the modified wedge method. (b) Data points with the same displacement from the velocity turning points were used to calculate the half-width $\left(W_{f}\right)$ and offset $\left(\Delta_{f}\right)$ of the lateral force loop.

\section{Shape factor}

To simplify the calculations, models of liquid bridges often reduce the complex geometries to easily solvable shapes, such as a cylinder [1], rectangular column [2] or, in this study, a decahedron. The ratio of the real to the predicted liquid bridge forces is often defined as the shape factor which reflects the topographical differences between the model and the exact shape of the liquid [3]. The shape factor of the lateral force, $K_{f}$, could be given by

$$
K_{f}=\frac{2 \Delta\left(F_{f} / \gamma_{L A}\left(w_{s}+w_{n}\right)\right)}{\Delta\left(\cos \theta_{l}-\cos \theta_{r}\right)} .
$$

Here, $F_{f}$ is the lateral force obtained by the experiment, $\gamma_{L A}$ is the liquid surface tension, $w_{s}$ and $w_{n}$ are the widths of the sliding surface and the neck, $\theta_{l}$ and $\theta_{r}$ are the left and right contact angles on the sliding surface. The results are shown in Fig. S4. 
The shape factor of the normal force, $K_{N}$, could be obtained from the ratio of the experimental value to the calculated result of Eq. 30 .

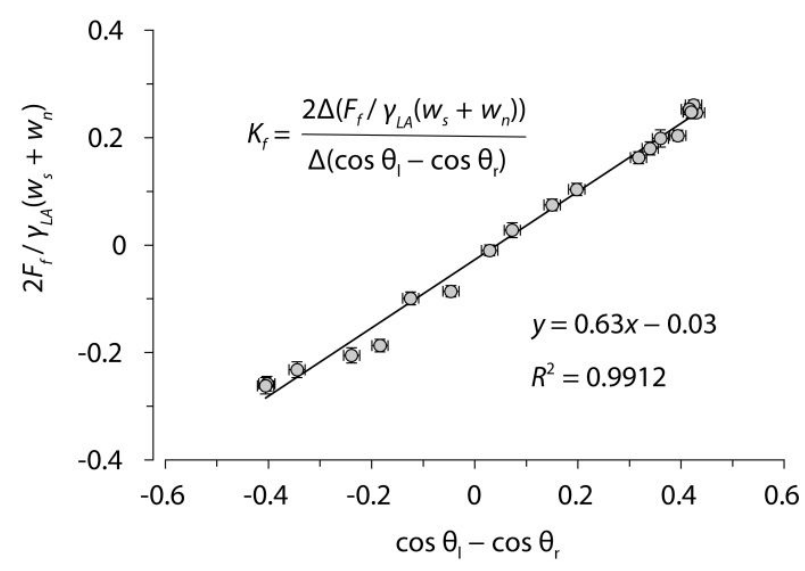

Fig. S4. The dimensionless lateral force of the water bridge plotted against the contact angle hysteresis. The slope of the curve was the shape factor of the lateral force.

\section{Experimental results for sliding occurring on the bottom plate}
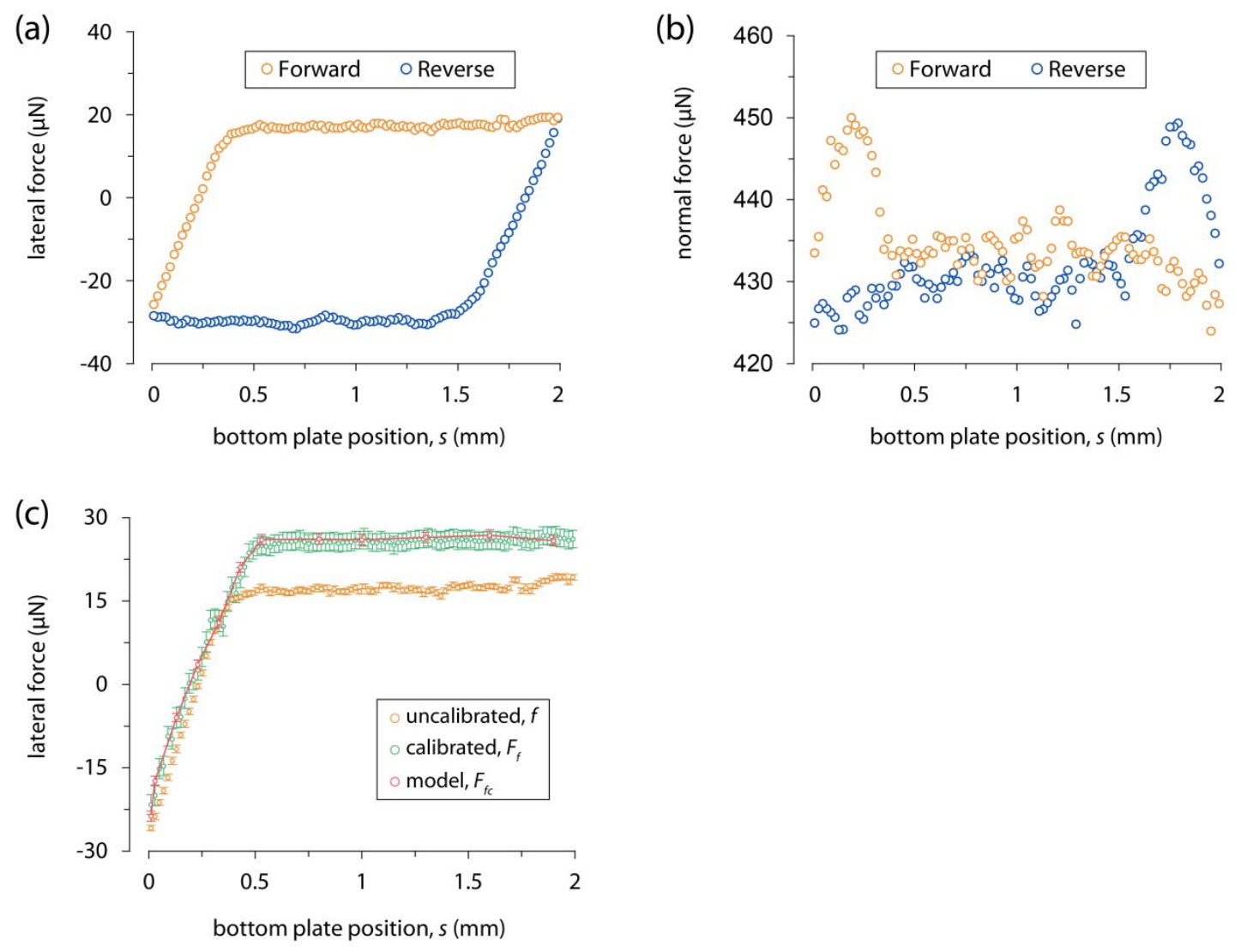
Fig. S5. Illustration of the capillary forces when the water bridge slid on the bottom plate. Uncalibrated (a) lateral and (b) normal adhesion forces of the water bridge plotted against the bottom plate position. (c) Uncalibrated, calibrated and calculated values of the lateral forces during the forward sliding. Error bars correspond to the measurement uncertainty calculated using the law of propagation of uncertainty. 


\section{References}

[1] M. Mastrangeli, J.-B. Valsamis, C. Van Hoof, J.-P. Celis, P. Lambert, Lateral capillary forces of cylindrical fluid menisci: a comprehensive quasi-static study, J. Micromech. Microeng. 20 (2010) 075041. https://doi.org/10.1088/0960-1317/20/7/075041.

[2] K. Tanaka, K. Iwamoto, Effective Shear Displacement on Lateral Adhesion Force of a Liquid Bridge Between Separated Plates, Tribol Lett. 64 (2016) 9. https://doi.org/10.1007/s11249-016-0744-1.

[3] A.I. ElSherbini, A.M. Jacobi, Retention forces and contact angles for critical liquid drops on non-horizontal surfaces, Journal of Colloid and Interface Science. 299 (2006) 841-849. https://doi.org/10.1016/j.jcis.2006.02.018. 